\title{
Role of charge fluctuation in Q1D organic superconductor $(\mathrm{TMTSF})_{2} \mathrm{ClO}_{4}$
}

\author{
Yusuke Mizuno $^{1}$, Akito Kobayashi ${ }^{1,2}$, and Yoshikazu Suzumura ${ }^{1}$ \\ ${ }^{1}$ Department of Physics, Nagoya University, Furo-cho, Chikusa-ku, Nagoya, 464-8602 \\ Japan \\ ${ }^{2}$ Institute for Advanced Research, Nagoya University, Furo-cho, Chikusa-ku, Nagoya, \\ 464-8602 Japan
}

\begin{abstract}
We have theoretically investigated the spin and charge fluctuations in the quasi-one dimensional organic superconductor (TMTSF) ${ }_{2} \mathrm{ClO}_{4}$. Using the extended multi-site Hubbard model, which contains four sites in a unit cell and the transfer energies obtained by the extended Hückel method, we calculate the linearized gap equation with the random phase approximation, to find novel order parameters of superconductivity due to several kinds of charge fluctuations induced by the anisotropic intersite repulsive interactions. For the singlet state, the order parameter with line nodes appears in the case of the strong charge fluctuation, while the order parameter with anisotropic gap suggested by Shimahara is reproduced in the spin fluctuation. The triplet state is also obtained for the wide parameter range of repulsive interactions due to a cooperation between charge and spin fluctuations.
\end{abstract}

Keywords: Organic Conductor, TMTSF, Superconductivity, Electron Correlation, Spin Fluctuation, Charge Fluctuation

\section{Introduction}

In quasi-one dimensional organic conductors TMTSF (tetrametyltetraselenafulvalence) salts, there are several experimental evidences of superconductivity, [1, 2, 3, 3, 4, 5, 6, 7, 8, 9, 10] which exhibit the large critical field being much larger than the Pauli limit. [5, 8, 9, 10] For (TMTSF) ${ }_{2} \mathrm{PF}_{6}$, the triplet superconducting(SC) state has been maintained from the measurement of the Knight shift and the NMR relaxation rate. [6, 7] For (TMTSF) ${ }_{2} \mathrm{ClO}_{4}$, 
[1] in the weak magnetic field, the singlet state with line nodes has been suggested by the the Knight shift and relaxation rate of the NMR experiment in the SC phase. [2, 4] Further, it has been suggested that the triplet or FFLO state emerges under strong magnetic field. [4, [8, 9, 10]

The specific property of (TMTSF) ${ }_{2} \mathrm{ClO}_{4}$ emerges due to the anion ordering below 24K. The folded Fermi surfaces with a unit cell containing four TMTSF molecules is obtained from the transfer integrals, which are estimated from the extended Hückel method based on the X-ray diffraction measurement.[11, 12, 13]

Several theoretical works have been performed to understand the superconductivity in the quasi-one dimensional organic conductors. The single band models for (TMTSF) ${ }_{2} \mathrm{PF}_{6}$ has been investigated by perturbation theories. [14, 15, 16, 17, 18, 19] The magnetic field favors the triplet or FFLO superconductivity, [16, 17, 18] while the on-site repulsion induces the singlet superconductivity in the absence of magnetic field. [19] The interplay between the spin and charge fluctuations in the triplet superconductivity has been investigated by the renormalization group theories. [20, 21, 22] The inter-site repulsions play important roles for the triplet superconductivity. [14, 15, 21, 22] In the multi band models for (TMTSF) ${ }_{2} \mathrm{ClO}_{4}[23$, 24], on the other hand, the singlet superconductivity with anisotropic gap (but without line nodes) has been suggested in the absence of magnetic field. [23]

In the present study, the possible singlet state with line nodes in the absence of magnetic field is investigated, since the experiment indicates the existence of line nodes. [8] We use the extended multi-site Hubbard model representing the system of (TMTSF) ${ }_{2} \mathrm{ClO}_{4}$, when the transfer energies are given by the extended Hückel method with the X-ray diffraction measurement.[13] The unit cell consists of four TMTSF sites owing to the presence of the anion ordering. Fruther, the roles of the charge fluctuation for the triplet state are also investigated on such a multi-site system. The spin and charge fluctuations are treated by the random phase approximation (RPA) on the site-representation, [25] and the order parameters of the superconductivity are evaluated using the linearized gap equation as described in $\S 2$. The singlet and triplet superconductivities are investigated by varying the magnitude of the anisotropic nearest-neighbor repulsive interactions with the fixed on-site interaction in $\S 3$. The origins and properties of those SC states are discussed in $\S 4$. In $\S 5$, summary and discussion are given. 


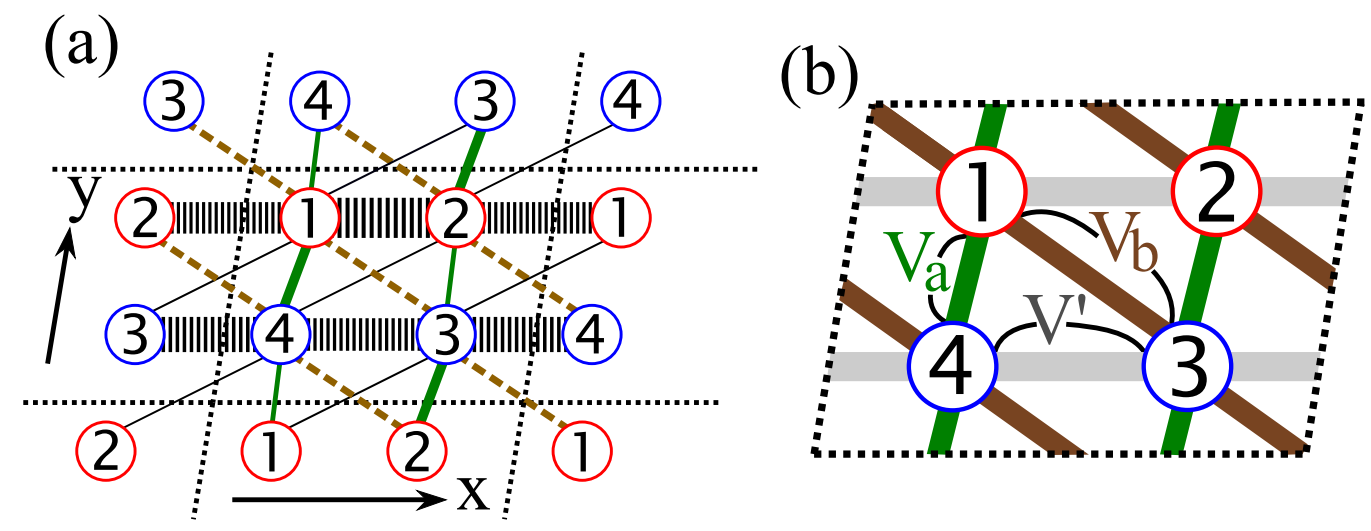

Figure 1: (a) A model describing the two-dimensional electronic system for (TMTSF) ${ }_{2} \mathrm{ClO}_{4}$. The $\mathrm{x}$-axis is taken as the stacking direction. The unit cell (the dotted line) consists of 4 molecules with several transfer energies $t_{\mathbf{d} ; \alpha \beta}$. (b) Inter-site repulsive interactions $V_{a}, V_{b}$ and $V^{\prime}$, where $V^{\prime}$ is discarded.

\section{Formulation}

\subsection{Model}

Figure 1 (a) shows the transfer integrals describing the two-dimensional (2D) electronic system for (TMTSF $)_{2} \mathrm{ClO}_{4}$, where we choose the x-axis along the stacking direction of TMTSF molecules. The thickness of the lines connecting between the molecules represents the relative magnitude of $t_{\mathbf{d} ; \alpha \beta}$. Figure 1 (b) displays the inter-site replusive interactions, which are taken in the present model.

Based on Figs. 1 (a) and (b), the extended multi-site Hubbard model is given by

$$
\mathcal{H}=\mathcal{H}_{0}+\mathcal{H}^{\prime}
$$

with

$$
\mathcal{H}_{0}=\sum_{\mathbf{i} \mathbf{d} \alpha \beta \sigma}\left(\left(t_{\mathbf{d} ; \alpha \beta}-\mu \delta_{\alpha, \beta} \delta(\mathbf{d})\right) a_{\mathbf{i} \alpha \sigma}^{\dagger} a_{\mathbf{i}+\mathbf{d} \beta \sigma}+\text { h.c. }\right)
$$

and

$$
\mathcal{H}^{\prime}=\sum_{\mathbf{i} \alpha} U a_{\mathbf{i} \alpha \uparrow}^{\dagger} a_{\mathbf{i} \alpha \downarrow}^{\dagger} a_{\mathbf{i} \alpha \downarrow} a_{\mathbf{i} \alpha \uparrow}+\sum_{\mathbf{i} \mathbf{d} \alpha \beta \sigma \sigma^{\prime}} V_{\mathbf{d} ; \alpha \beta} a_{\mathbf{i} \alpha \sigma}^{\dagger} a_{\mathbf{i}+\mathbf{d} \beta \sigma^{\prime}}^{\dagger} a_{\mathbf{i}+\mathbf{d} \beta \sigma^{\prime}} a_{\mathbf{i} \alpha \sigma},
$$


where $\mathbf{i}\left(=(1,1) \cdots\left(\sqrt{N_{L}}, \sqrt{N_{L}}\right)\right)$ denotes a two dimensional lattice vector for the unit cell, and $\mathbf{d}$ is a vector connecting different cells. Quantities $\alpha$ and $\beta(=1,2,3,4)$ are indices of molecules in the unit cell and $\sigma(=\uparrow, \downarrow)$ represent spin. The transfer energies, $t_{\mathbf{d} ; \alpha \beta}$, are obtained by the extended Hückel method based on the X-ray diffraction measurement. [12, 13, 11] Coupling constants $U$ and $V_{\mathbf{d} ; \alpha \beta}$ correspond to the on-site and nearest neighbor Coulomb interactions, respectively. For $V_{\mathbf{d} ; \alpha \beta}$, there is a relation, $V_{\mathbf{d} ; \alpha \beta}=$ $V_{\mathbf{d} ; \beta \alpha}=V_{-\mathbf{d} ; \alpha \beta}$. The unit of the energy is taken as $\mathrm{eV}$.

We investigate the superconductivity by varying the nearest neighbor Coulomb interactions between the TMTSF chains, i.e., $V_{a}$ along the $y$ axis and $V_{b}$ along the $x+y$ axis (see Fig. 1 (b)). In the RPA, the critical value of the nearest neighbor Coulomb interaction along the intrachain, $V^{\prime}$, at which the charge fluctuation diverges, is more than twice of those of $V_{a}$ and $V_{b}$. This comes from the fact that the role of the nesting vector is incompatible with that of $V^{\prime}$. The same fact has been shown in the renormalization group theory. 22] Thus $V^{\prime}$ is discarded in the present study.

Using Fourier transformation, the Hamiltonian is rewritten as

$$
\begin{aligned}
\mathcal{H} & =\sum_{\mathbf{k} \sigma \alpha \beta} \epsilon_{\alpha \beta}(\mathbf{k}) a_{\mathbf{k} \sigma \alpha}^{\dagger} a_{\mathbf{k} \sigma \beta} \\
& +\frac{1}{N} \sum_{\mathbf{k} \mathbf{k}^{\prime} \mathbf{q} \alpha} U a_{\mathbf{k}+\mathbf{q} \uparrow \alpha}^{\dagger} a_{\mathbf{k}^{\prime}-\mathbf{q} \downarrow \alpha}^{\dagger} a_{\mathbf{k}^{\prime} \downarrow \alpha} a_{\mathbf{k} \uparrow \alpha} \\
& +\frac{1}{2 N} \sum_{\mathbf{k} \mathbf{k}^{\prime} \mathbf{q} \sigma \sigma^{\prime} \alpha \beta} V_{\alpha \beta}(\mathbf{q}) a_{\mathbf{k}+\mathbf{q} \sigma \alpha}^{\dagger} a_{\mathbf{k}^{\prime}-\mathbf{q} \sigma^{\prime} \beta}^{\dagger} a_{\mathbf{k}^{\prime} \sigma^{\prime} \beta} a_{\mathbf{k} \sigma \alpha}
\end{aligned}
$$

where

$$
\begin{aligned}
\epsilon_{12}(\mathbf{k}) & =t_{12}+t_{12}^{\prime} e^{i k_{x}} \\
\epsilon_{13}(\mathbf{k}) & =t_{13}+t_{13}^{\prime}\left(e^{i k_{x}}+e^{-i k_{y}}\right)+t_{13}^{\prime \prime} e^{i\left(k_{x}-k_{y}\right)} \\
\epsilon_{14}(\mathbf{k}) & =t_{14}+t_{14}^{\prime} e^{-i k_{y}} \\
\epsilon_{23}(\mathbf{k}) & =t_{23}+t_{23}^{\prime} e^{-i k_{y}} \\
\epsilon_{24}(\mathbf{k}) & =t_{24}+t_{24}^{\prime}\left(e^{-i k_{x}}+e^{-i k_{y}}\right)+t_{24}^{\prime \prime} e^{-i\left(k_{x}+k_{y}\right)} \\
\epsilon_{34}(\mathbf{k}) & =t_{34}+t_{34}^{\prime} e^{-i k_{x}} \\
V_{13}(\mathbf{q}) & =V_{13}+V_{13}^{\prime}\left(e^{i q_{x}}+e^{-i q_{y}}\right)+V_{13}^{\prime \prime} e^{i\left(q_{x}-q_{y}\right)} \\
V_{14}(\mathbf{q}) & =V_{14}+V_{14}^{\prime} e^{-i q_{y}}
\end{aligned}
$$




$$
\begin{aligned}
& V_{23}(\mathbf{q})=V_{23}+V_{23}^{\prime} e^{-i q_{y}}, \\
& V_{24}(\mathbf{q})=V_{24}+V_{24}^{\prime}\left(e^{-i q_{x}}+e^{-i q_{y}}\right)+V_{24}^{\prime \prime} e^{-i\left(q_{x}+q_{y}\right)},
\end{aligned}
$$

with $\epsilon_{\beta \alpha}=\epsilon_{\alpha \beta}^{*}, V_{\beta \alpha}=V_{\alpha \beta}^{*}$, and the other elements are zero. The transfer energies are given by [12, 13, 11] $t_{12}=0.413, t_{12}^{\prime}=0.324, t_{34}=0.335$ and $t_{34}^{\prime}=0.362$ along conduction chains, $t_{23}=t_{14}^{\prime}=-0.050, t_{14}=t_{23}^{\prime}=-0.100$ along y-axis, and $t_{24}^{\prime}=0.070, t_{24}=t^{\prime \prime}{ }_{24}=0.020, t_{13}=t{ }_{13}=0.071, t_{13}^{\prime}=$ 0.021 where $t, t^{\prime}$ and $t^{\prime \prime}$ correspond to transfer energies between molecules in a unit cell, those between the nearest neighbor cells, and those between the next-nearest neighbor cells, respectively. Note $t_{\alpha \beta}=t_{\beta \alpha}$. We choose $V_{14}=$ $V_{23}=V_{14}^{\prime}=V_{23}^{\prime}=V_{a}$ for nearest neighbor, i,e, $\mathbf{d}=( \pm 1,0),(0, \pm 1)$ and $V_{13}=$ $V_{24}^{\prime}=V_{24}^{\prime}=V_{13}^{\prime \prime}=V_{b}$ for next nearest neighbor, i.e., $\mathbf{d}=( \pm 1, \pm 1),( \pm 1, \mp 1)$. (See Fig. 1(b).) The tight-binding Hamiltonian Eq. (2) is diagonalized by

$$
\sum_{\beta}^{4}\left(\epsilon_{\alpha \beta}(\mathbf{k})-\mu \delta_{\alpha \beta}\right) d_{\beta}^{A}(\mathbf{k})=\xi_{\mathbf{k}}^{A} d_{\alpha}^{A}(\mathbf{k}),
$$

where $A(=1,2,3,4)$ is an index of the band representation and $\xi^{A}(\mathbf{k})$ denotes the eigen energy measured from the Fermi energy $(=\mu)$. note that the eigenvector $d_{\beta}^{A}(\mathbf{k})$ gives the transformation from the site representation to the band representation.

In Fig. 2(a), the energy band spectrum is shown. There are four bands due to four molecules in the unit cell where the first and second bands from the top crosses the Fermi surface. The shape of quasi one-dimensional Fermi surface is shown in Fig. 2(b) representing the folded Fermi surface. In Fig. 3. the density of states, $D(\epsilon)$, is shown where

$$
D(\epsilon)=-\frac{1}{N \pi} \sum_{\mathbf{k} \sigma A} \operatorname{Im}\left(\frac{1}{\epsilon+i \delta-\xi_{\mathbf{k}}^{A}+\mu}\right),
$$

and sum rule is given by $\int D(\epsilon) d \epsilon=8$. These double one-dimensional Fermi surfaces have been observed by AMRO[11].

\subsection{Pairing Interactions and Gap Equation}

The spin and charge fluctuations in the weak coupling regime are treated by RPA. [14, 15, 16, 17, 19, 26, 25] where the site representation [26] for singlet and triplet pairing interactions are given respectively as

$$
\hat{P}^{\text {Singlet }}=\hat{U}+\hat{V}+\frac{3}{2} \hat{U} \hat{\chi}^{S} \hat{U}-\frac{1}{2}(\hat{U}+2 \hat{V}) \hat{\chi}^{C}(\hat{U}+2 \hat{V})
$$




$$
\hat{P}^{\text {Triplet }}=\hat{V}-\frac{1}{2} \hat{U} \hat{\chi}^{S} \hat{U}-\frac{1}{2}(\hat{U}+2 \hat{V}) \hat{\chi}^{C}(\hat{U}+2 \hat{V}) .
$$

The hat denotes a matrix representation with an element $(\hat{f})_{\alpha \beta}=f_{\alpha \beta}$. The pairing interactions are obtained from $\hat{\chi}^{S}$ and $\hat{\chi}^{C}$ which denote spin susceptibility and charge susceptibility;

$$
\hat{\chi}^{S}=\left(\hat{I}-\hat{\chi}^{0} \hat{U}\right)^{-1} \hat{\chi}^{0}
$$

and

$$
\hat{\chi}^{C}=\left(\hat{I}+2 \hat{\chi}^{0} \hat{V}+\hat{\chi}^{0} \hat{U}\right)^{-1} \hat{\chi}^{0} .
$$

The quantity $\hat{\chi}^{0}$ is an irreducible susceptibility which is given by

$$
\begin{aligned}
\left(\hat{\chi}^{0}(\mathbf{q})\right)_{\alpha \beta} & =\frac{-1}{N_{L}} \sum_{A B \mathbf{k}} d_{\alpha}^{A}(\mathbf{k}+\mathbf{q}) d_{\beta}^{* A}(\mathbf{k}+\mathbf{q}) d_{\alpha}^{* B}(\mathbf{k}) d_{\beta}^{B}(\mathbf{k}) \\
& \times \frac{f\left(\xi_{\mathbf{k}+\mathbf{q}}^{A}\right)-f\left(\xi_{\mathbf{k}}^{B}\right)}{\xi_{\mathbf{k}+\mathbf{q}}^{A}-\xi_{\mathbf{k}}^{B}}
\end{aligned}
$$

where $f(x)=\left(e^{x / T}+1\right)^{-1}$ is the Fermi distribution and $T$ denotes temperature.

In order to examine a symmetry of order parameter of the superconductivity, we solve numerically a linearized gap equation given by,

$$
\lambda \Sigma_{\alpha \beta}^{a}(\mathbf{k})=-\frac{T}{N_{L}} \sum_{\mathbf{k}^{\prime} \alpha^{\prime} \beta^{\prime}}\left[\left(\hat{P}^{A}\left(\mathbf{k}-\mathbf{k}^{\prime}\right)\right)_{\alpha \beta} G_{\alpha \alpha^{\prime}}^{0}\left(\mathbf{k}^{\prime}\right) G_{\beta \beta^{\prime}}^{0}\left(-\mathbf{k}^{\prime}\right)\right] \Sigma_{\alpha \beta}^{a}\left(\mathbf{k}^{\prime}\right) .
$$

$\Sigma_{\alpha \beta}^{a}(\mathbf{k})$ is anomalous self energy and $G_{\alpha \alpha^{\prime}}^{0}\left(\mathbf{k}^{\prime}\right)$ denotes a bare Green's function written as

$$
G_{\alpha \beta}^{0}\left(\mathbf{k}, i \varepsilon_{n}\right)=\sum_{A} d_{\alpha}^{A}(\mathbf{k}) d_{\beta}^{* A}(\mathbf{k}) \frac{1}{i \varepsilon_{n}-\xi_{\mathbf{k}}^{A}},
$$

where $\varepsilon_{n}=(2 n+1) \pi T$ is Matsubara-frequency $\left(k_{B}=1\right)$ and $\mathrm{n}$ is an integer. The quantity $\hat{P}^{A}$ (A = Singlet, Triplet) denotes the paring interactions for singlet state and triplet state which are rewritten as

$$
\hat{P}^{\text {Singlet }}=\hat{P}^{S_{S}}+\hat{P}^{C},
$$

and

$$
\hat{P}^{\text {Triplet }}=\hat{P}^{S_{T}}+\hat{P}^{C} .
$$


The r.h.s. of Eqs. (16) and (17) is given by

$$
\begin{gathered}
\hat{P}^{S_{S}}=\hat{U}+\frac{3}{2} \hat{U} \hat{\chi}^{S} \hat{U} \\
\hat{P}^{S_{T}}=-\frac{1}{2} \hat{U} \hat{\chi}^{S} \hat{U} \\
\hat{P}^{C}=\hat{V}-\frac{1}{2}(\hat{U}+2 \hat{V}) \hat{\chi}^{C}(\hat{U}+2 \hat{V}) .
\end{gathered}
$$

Note that $\hat{P}^{S_{S}}$ and $\hat{P}^{S_{T}}$ represent spin fluctuation, and $\hat{P}^{C}$ denotes the charge fluctuation.

The transition temperature for the $\mathrm{SC}$ state, $T_{\mathrm{c}}$, is determined by the condition, $\lambda=1$. The gap function at $T=0, \Delta_{\alpha \beta}(\mathbf{k})$, is estimated from $\Delta_{\alpha \beta}(\mathbf{k})=g \Sigma_{\alpha \beta}^{a}(\mathbf{k})$, where $g$ is chosen to reproduce the gap which is expected from $T_{\mathrm{c}}$. [26] Using $\Delta_{\alpha \beta}(\mathbf{k})$, we get the quasi-particle bands $E_{\mathbf{k}}^{A^{\prime}}$ by diagonalizing Hamiltonian $\mathcal{H}$,

$$
\begin{aligned}
& \mathcal{H}=\sum_{\mathbf{k} \alpha \beta \sigma} \epsilon_{\alpha \beta}(\mathbf{k}) a_{\mathbf{k} \alpha \sigma}^{\dagger} a_{\mathbf{k} \beta \sigma} \\
&-\sum_{\mathbf{k} \alpha \beta} \Delta_{\alpha \beta}(\mathbf{k}) a_{\mathbf{k} \alpha \uparrow}^{\dagger} a_{-\mathbf{k} \beta \downarrow}^{\dagger} \\
&-\sum_{\mathbf{k} \alpha \beta} \Delta_{\alpha \beta}^{*}(\mathbf{k}) a_{-\mathbf{k} \beta \downarrow} a_{\mathbf{k} \alpha \uparrow}, \\
& \sum_{\beta^{\prime}}^{8} \tilde{\epsilon}_{\alpha^{\prime} \beta^{\prime}}(\mathbf{k}) d_{\beta^{\prime}}^{A^{\prime}}(\mathbf{k})=E_{\mathbf{k}}^{A^{\prime}} d_{\alpha^{\prime}}^{A^{\prime}}(\mathbf{k}),
\end{aligned}
$$

where

$$
\tilde{\epsilon}(\mathbf{k})=\left(\begin{array}{cc}
\hat{\epsilon}(\mathbf{k})-\mu \delta_{\alpha \beta} & -\hat{\Delta}(\mathbf{k}) \\
-\hat{\Delta}^{\dagger}(-\mathbf{k}) & -{ }^{t} \hat{\epsilon}(-\mathbf{k})+\mu \delta_{\alpha \beta}
\end{array}\right)
$$

\section{Singlet SC state vs. triplet SC state}

\subsection{Band Calculation and Model Setting}

In the present study temperature is fixed at $\mathrm{T}=0.01[\mathrm{eV}]$. We take $24 \times$ 24 meshes for calculating the bands, the susceptibilities, and the superconductivity. The energy bands are shown in Fig. 2(a). Since the electrons are 
(a)

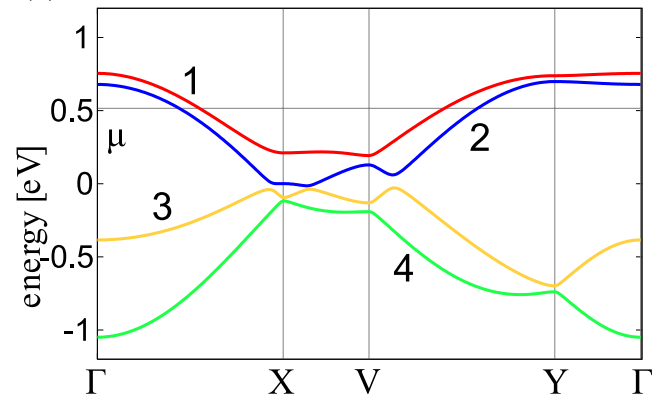

(b)

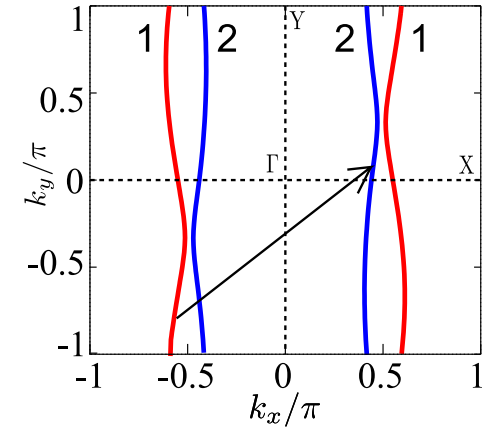

Figure 2: (a) Energy Band for (TMTSF) ${ }_{2} \mathrm{ClO}_{4}$ given by Eq. (2) with the $3 / 4$ filling, where the two bands 1 and 2 from the top cross the Fermi energy, $\mu$. (b) The Fermi surfaces for the first and second bands, where the arrow represents the nesting vector.

3/4-filled, the Fermi surfaces exist in upper two bands. The density of states (DOS) is shown in Fig. 3, There is a large Van Hove singularity above the Fermi energy.

Now we consider the electron correlation effects. It is expected that $U$ plays important roles for superconductivity in (TMTSF) ${ }_{2} \mathrm{ClO}_{4}$, because (TMTSF $)_{2} \mathrm{ClO}_{4}$ is located next to the spin density wave (SDW) state in the phase diagram suggested by Jerome. [3] Then we calculate the possible SC states in (TMTSF $)_{2} \mathrm{ClO}_{4}$ by choosing $U=0.60$ which is close to the critical value for the divergence of the spin fluctuation in the RPA. We investigate the roles of the charge fluctuation by varying the interchain repulsive interactions, $V_{a}$ and $V_{b}$, with the fixed $U$, where we define $V$ as

$$
V \equiv \sqrt{V_{a}^{2}+V_{b}^{2}}
$$

\subsection{Superconductivity for $V=0$}

For $V_{a}=V_{b}=0$, spin and charge pairing interactions $\left(\hat{P}^{S}\right.$ and $\left.\hat{P}^{C}\right)$ are shown in Fig. 4 where $\hat{P}^{S}$ is much larger than $\hat{P}^{C}$. The susceptibility $\hat{\chi}^{S}$ which determines $\hat{P}^{S}$ is strongly enhanced by $U$ compared with $\hat{\chi}^{C}$, since the $\mathbf{q}$ vector for the peak of $\hat{\chi}^{S}$ is the same as the nesting vector.

Now we examine numerically Eq. (14) to find that $\lambda$ for spin singlet is larger than that for spin triplet, e.g., $\lambda=0.9$ for the singlet state and 0.3 


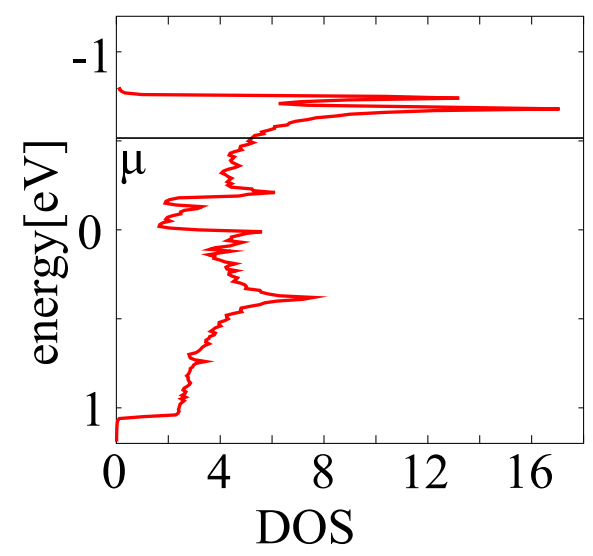

Figure 3: The density of states (horizontal axis).

for the triplet state at $U=0.6$ and $V_{a}=V_{b}=0$. In the present paper, a phase factor in anomalous self energy is determined in order to maximize the value $\sum_{\alpha \beta \mathbf{k}}\left|\operatorname{Re} \Sigma_{\alpha \beta}^{a}(\mathbf{k})\right|$. The anomalous self energy consists of 16 elements of $(\alpha, \beta)$ on the basis of site representation. Diagonal elements $(\alpha, \alpha)$ are larger than others. The momentum dependence of these elements is given by " $\cos k_{x}$ " which indicates a sign change on a line separating two Fermi surfaces in Fig. 2(b). The absence of the node on the Fermi surface, which gives a full gap state (written as "singlet (full)"), is consistent with that of Shimahara's result. [23] As for the off-diagonal element of $\Sigma_{\alpha \beta}^{a}(\mathbf{k})$, there is a clear contribution from the same chain , e.g., $(1,2)$ and $(3,4)$, while that from the interchain is negligibly small. These behavior of intrachain dependence suggests a role of dimerization, which reduces to the half-filled band as shown in Fig. 2,

\subsection{Superconductivity for $V \neq 0$}

We examine the effect of inter chain interactions $V$ to find a possibility of new $\mathrm{SC}$ state which is induced by charge fluctuation. With increasing $V$, $\hat{\chi}^{C}$ increases, and diverges at $V=V_{C}$. In order to calculate the region where the charge fluctuation becomes a dominant one, $V$ is chosen to satisfy the condition that $\hat{\chi}^{C}>3 \hat{\chi}^{S}$ and $V<V_{C}$. We examine the SC state on the line from $\left(V_{a}, V_{b}\right)=(0,0)(\mathrm{O})$ to several points given by $\left(V_{a}, V_{b}\right)=(0.00,0.64)$ (a), $(0.11,0.64)(\mathrm{b}),(0.21,0.64)(\mathrm{c}),(0.33,0.62)(\mathrm{d}),(0.49,0.49)(\mathrm{e}),(0.67,0.22)(\mathrm{f})$ and $(0.70,0.00)(\mathrm{g})$, respectively. The ratio of $V_{a}$ to $V_{b}$ increases as the point 

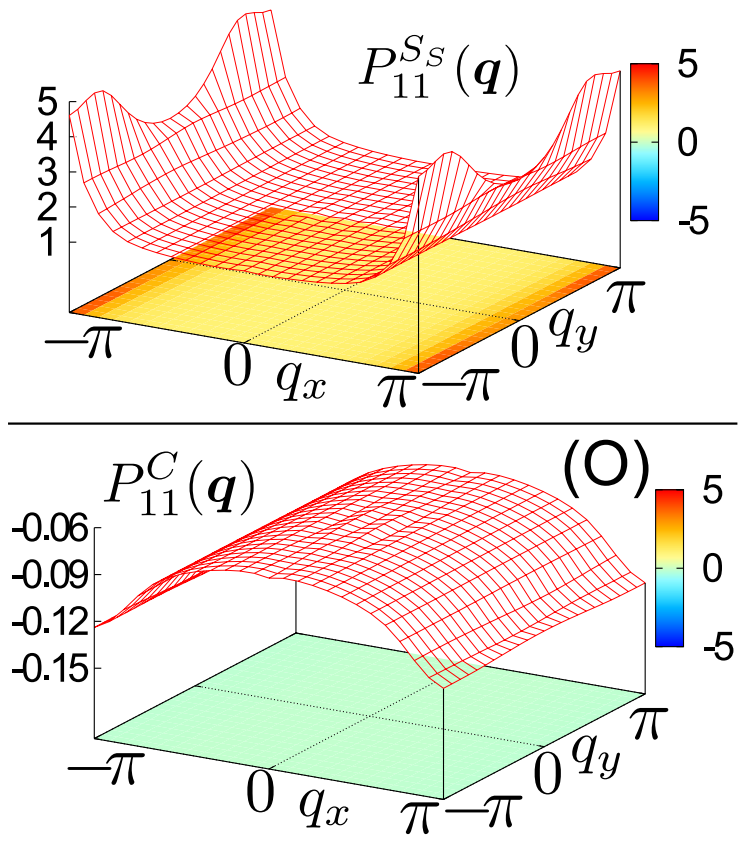

Figure 4: The momentum dependence of pairing interactions for the elements $\hat{P}_{11}^{S}$ and $\hat{P}_{11}^{C}$ at $T=0.01$ and $V=0$. The red and blue represent the repulsive and attractive interactions, respectively. The symbol $\mathrm{O}$ corresponds to $V_{a}=V_{b}=0$.

moves from (a) to (g). By solving Eq. (14), $\lambda$ for singlet and triplet states are calculated and the larger one is chosen as the expected state.

In the region of small $V$, the singlet $\mathrm{SC}$ state similar to one at $V=0$ is realized where the state with a full gap is found. In Fig. 5, the $V$ dependence of $\lambda$ of both singlet and triplet states is shown as a function of $V$ for three cases of $(\mathrm{O}) \rightarrow(\mathrm{a}),(\mathrm{O}) \rightarrow(\mathrm{d})$, and $(\mathrm{O}) \rightarrow(\mathrm{g})$. For the variation from $(\mathrm{O})$ to (a), (the first case), the singlet state is suppressed and the triplet state is expected. For the variation of $V$ from $(\mathrm{O})$ to $(\mathrm{d})$ (the second case), the singlet state moves to the triplet state close to $V=V_{C}$. For the variation of $V$ from (O) to (g) (the third case), the singlet state remains as the dominant state but moves to another singlet state (as explained later) after $\lambda$ takes a minimum close to $(\mathrm{g})$.

Here we note the $\mathrm{V}$ dependence of $\lambda$ for triplet state, which is enhanced by $\hat{\chi}^{C}$. From Eq. (10), the term for the spin fluctuation and that for the charge fluctuation has the same sign in triplet pairing interaction. Although 


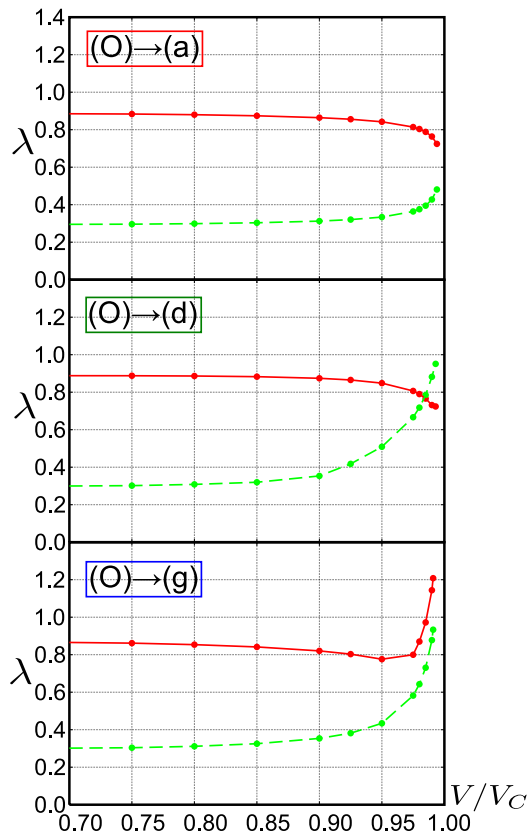

Figure 5: $\quad \mathrm{V}$ dependence of $\lambda$ for singlet and triplet states for $T=0.01, U=0.6$. The horizontal axis is shown for $V / V_{C}>0.7$ which represents from the point (O) to (a), from the point $(\mathrm{O})$ to $(\mathrm{d})$ and from the point $(\mathrm{O})$ to $(\mathrm{g})$, where these points in the parameter space are given by $\left(V_{a}, V_{b}\right)=(0,0)(\mathrm{O}),(0.00,0.64)(\mathrm{a}),(0.33,0.62)(\mathrm{d})$, and $(0.70,0.00)(\mathrm{g})$, respectively. The solid line denotes $\lambda$ for the singlet state and the dashed line denotes $\lambda$ for the triplet state.

the triplet SC state is enhanced but the singlet state is suppressed by charge fluctuations, such an effect becomes large only close to $V=V_{C}$. This is why the singlet state is favored and the region for the triplet state is small even in the presence of $V$.

\section{Pairing interaction and Anomalous self-energy}

Now we examine the respective SC state precisely based on the pairing interaction shown in Figs. 6 and anomalous self-energy.

(i) First, we discuss the case indicating a possible triplet SC state, which is given by (a) and (d). When $\hat{\chi}^{C}$ is increased by $V$, the singlet state is suppressed but the triplet state is enhanced (Fig. 6 (a) and (d)). Actually, the competition between these two kinds of fluctuations occurs for the singlet 


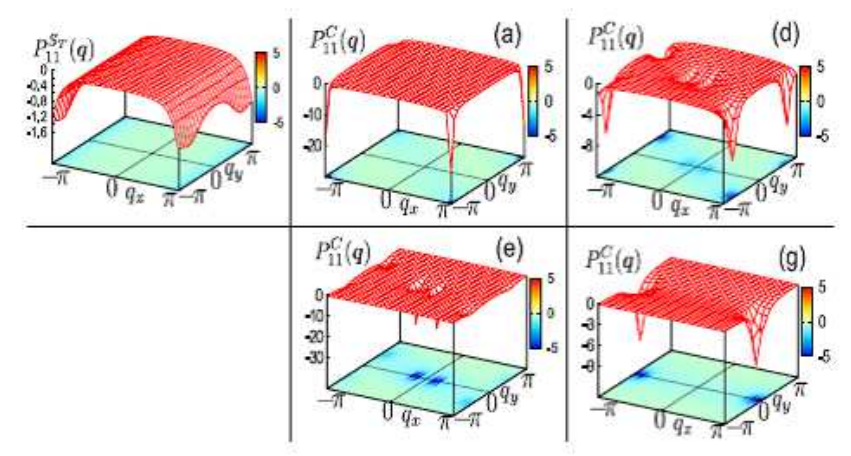

Figure 6: The momentum dependence of charge-fluctuation induced pairing interaction $\left(\hat{P}_{11}^{C}\right)$ for $V \neq 0$. where parameters corresponding to points (a), (d), (e) and (g) are $(0.00,0.64),(0.33,0.62),(0.49,0.49)$, and $(0.70,0.00)$, respectively. The attractive interaction is represented by blue on the $k_{x}-k_{y}$ plane. For the comparison, $P_{11}^{S_{T}}$ is shown.

state as seen from Eq. (9) and by noting a fact that the peak position of $\hat{P}^{C}$ is nearly the same as that of $\hat{P}_{S}^{S}$ from Fig. 4. The triplet state may be expected when $V \rightarrow V_{C}$, and is rather favored by the introduction of $V_{a}$. When the point moves from (a) to (d) (i.e., increasing $V_{a}$ ), the peak position of $\hat{P}^{C}$ begins to deviate slightly from $(\pi, \pi)$ and the attractive interaction increases due to the increase of the amount of $\hat{P}^{C}$ integrated with respect to the momentum.

(ii) Secondly, we study the region where the singlet state is favored by the effects of $\hat{\chi}^{C}$ (points (e) - (g). The pairing interactions $\left(\hat{P}^{C}\right)$ induced by charge fluctuation are shown in Figs. 66 (e) and (g) where the position of the peak is different from that of in $\hat{\chi}^{S}$. As a result, the effect of $\hat{\chi}^{C}$ on the singlet pairing state becomes compatible with that of $\hat{\chi}^{S}$ for $V \rightarrow V_{c}$. Further since $\hat{P}^{C}$ is larger than $\hat{P}^{S}$, the spin singlet state is determined mainly by $\hat{\chi}^{C}$ and is assisted by $\hat{\chi}^{S}$. The change of the self-energy along $q_{x}$ axis is very small. This comes from the difference in the peak position of charge fluctuations i.e., $(\pi, \pi)$ for (a) and that on the $q_{x}$ axis for (e) and (g).

Finally, we examine the node of the gap, which is calculated from Eqs. (21) and (22). Figure 7 denotes the position of nodes and Fermi surfaces for points $(\mathrm{O}),(\mathrm{f}),(\mathrm{g})$ and $(\mathrm{d})$. For the singlet state $(\mathrm{O})$, which is mediated by $\hat{\chi}^{S}$ the node exists between two different Fermi surfaces implying a full gap due to the existence of the two bands. [23] For (f) and (g), the singlet state mediated by $\hat{\chi}^{C}$ has two nodes at $k_{y}= \pm \pi / 2$, which cross the Fermi surfaces. 

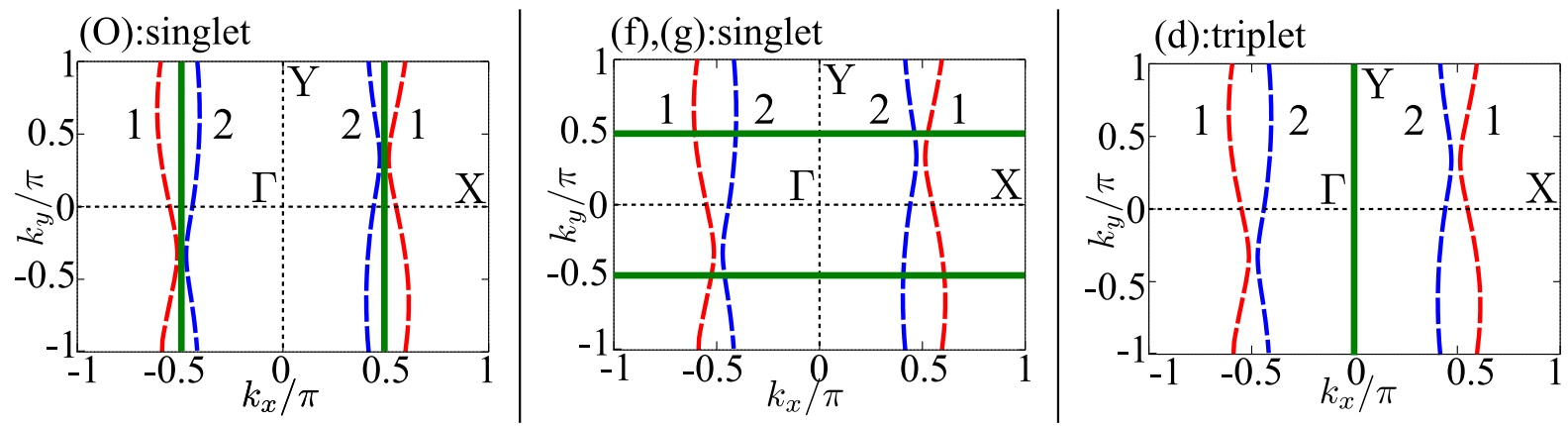

Figure 7: Fermi surface and gap node where the dashed lines denotes Fermi surface and the solid line denote gap node at $T=0.01, U=0.6$.

Gap function for triplet has a node on the line $k_{x}=0$ suggesting a full-gap state.

\section{Summary and Discussion}

We have found the possible singlet SC state with the line nodes in the extended multi-site Hubbard model representing (TMTSF) ${ }_{2} \mathrm{ClO}_{4}$ with the multi-band and under the anion ordering. Such a state is induced by the charge fluctuation owing to the interchain Coulomb interaction $V_{a}$ (along the $y$-axis), while the singlet SC state with anisotropic (but finite) gap suggested by Shimahara [23] is reproduced in the presence of the strong spin fluctuation. Moreover, we have investigated the roles of the charge fluctuation for the triplet SC state. It is found that the triplet state appears in a certain range of the interchain Coulomb interactions, $V_{a}$ and $V_{b}$, It is noted that the charge fluctuation cooperates with the spin fluctuation for the triplet pairing, but competes for the singlet pairing. For such a triplet state, the p-wave like state is obtained in the present multi-site model, while the f-wave gap function have been suggested in the single site models. [14, 15, 16, 17, 18, 20, 21, 22]

Finally we discuss how to distinguish the SC state with the lines and that with full gap of anisotropy on the Fermi surface. Using a maximum, $\Delta_{\max }$, and a minimum, $\Delta_{\min }$, for the latter case, $1 / T_{1}$ reveals the exponential behavior at low temperature, i.e., $T<\Delta_{\min }$. In the preset case, it is expected that $1 / T_{1}$ exhibits exponential decrease at lower temperatures than $T_{\mathrm{c}} \Delta_{\min } / \Delta_{\max } \sim 0.2 \mathrm{~K}$ using the ratio given by both our and Shimahara's results. On the other hand, Yonezawa et al. have reported that the $1 / T_{1}$ 
exhibits $T^{3}$ behavior at temperatures lower than $0.2 \mathrm{~K}[8]$. This fact suggests that the SC state with the line nodes supports the result of the NMR experiment in the absence of magnetic field.

\section{Acknowledgment}

We are thank to S. Yonezawa, Y. Maeno, H. Shimahara for useful discussions. This work was financially supported in part by a Grant-in-Aid for Special Coordination Funds for Promoting Science and Technology (SCF), Scientific Research on Innovative Areas 20110002, and Scientific Research 19740205 from the Ministry of Education, Culture, Sports, Science and Technology of Japan.

\section{References}

[1] K. Bechgaard, K. Carneiro, M. Olsen, F. B. Rasmussen, and C. S. Jacobsen 1981 Phys. Rev. Lett. 46852.

[2] M. Takigawa, H. Yasuoka, and G. Saito 1987 J. Phys. Soc. Jpn. 56 873-876.

[3] D. Jérome 1991 Sciense 2521509.

[4] J. Shinagawa, Y. Kurosaki, F. Zhang, S. E. Brown, D. Jérome, J. B. Christensen, and K. Bechgaard 2007 Phys. Rev. Lett. 98147002.

[5] I. J. Lee, M. J. Naughton, G. M. Danner and P. M. Chaikin 1997 Phys. Rev. Lett. 783555.

[6] I. J. Lee, S. E. Brown, W. G. Clark, M. J. Strouse, M. J. Naughton, W. Kang, and P. M. Chaikin 2002 Phys. Rev. Lett. 88017004.

[7] I. J. Lee, D. S. Chow, W. G. Clark, M. J. Strouse, M. J. Naughton, P. M. Chaikin, and S. E. Brown 2003 Phys. Rev. B 68092510.

[8] S. Yonezawa, S. Kusaba, Y. Maeno, P. Auban-Senzier, C. Pasquier, K. Bechgaard, and D. Jérome 2008 Phys. Rev. Lett. 100117002.

[9] S. Yonezawa, S. Kusaba, Y. Maeno, P. Auban-Senzier, C. Pasquier, and D. Jérome 2008 J. Phys. Soc. Jpn. 77054712. 
[10] S. Kusaba, S. Yonezawa, Y. Maeno, P. Auban-Senzier, C. Pasquier, K. Bechgaard, D. Jérome 2008 Solid State Science 10 1768-1772.

[11] H. Yoshino, A. Oda, T. Sakaki, T. Hanajiri, J. Yamada, S. Nakatsuji, H. Anzai and K. Murata 1999 J. Phys. Soc. Jpn. 683142.

[12] J. P. Pouget, G. Shirane, K. Bechgaard and J. M. Fabre 1983 Phys. Rev. B 275203.

[13] D. Le Pévelen, J. Gaultier, Y. Barrans, D. Chasseau, F. Castet, and L. Ducasse 2001 The European Physical Journal B 19363.

[14] Y. Tanaka, and K. Kuroki 2004 Phys. Rev. B 70060502.

[15] K. Kuroki, and Y. Tanaka 2005 J. Phys. Soc. Jpn. 741694.

[16] H. Aizawa, K. Kuroki, and Y. Tanaka 2008 Phys. Rev. B 77144513.

[17] H. Aizawa, K. Kuroki, T. Yokoyama, and Y. Tanaka 2009 Phys. Rev. Lett. 102016403.

[18] N. Belmechri, G. Abramovici, and M. Heritier 2008 Eur. Phys. Lett. 82 47009 .

[19] H. Kino, and H. Kontani 1999 J. Phys. Soc. Jpn. 681481.

[20] R. Duprat, and C. Bourbonnais 2001 Eur. Phys. J. B. 21219.

[21] Y. Fuseya, and Y. Suzumura 2005 J. Phys. Soc. Jpn. 741263.

[22] J. C. Nickel, R. Duprat, C. Bourbonnais, and N. Dupuis 2005 Phys. Rev. Lett. 95247001.

[23] H. Shimahara 2000 Phys. Rev. B 6114936.

[24] Y. Hasegawa and K. Kishigi 2008 Phys. Rev. B 78045117.

[25] A. Kobayashi, Y. Tanaka, M. Ogata, and Y. Suzumura 2004 J. Phys. Soc. Jpn. 731115.

[26] A. Kobayashi, S. Katayama, K. Noguchi, and Y. Suzumura 2004 J. Phys. Soc. Jpn. 733135. 